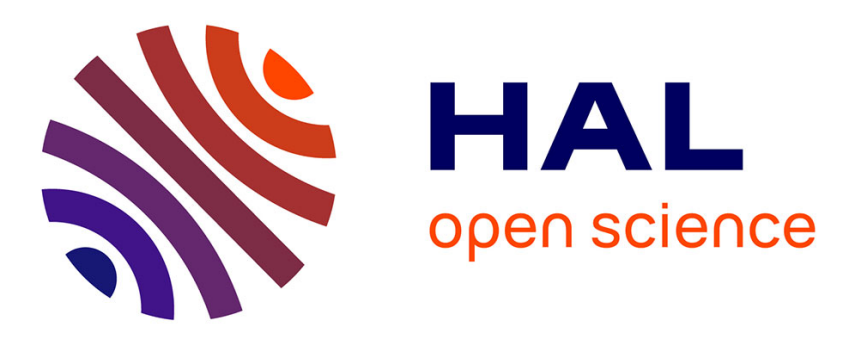

\title{
Global stability analysis of flow through a fusiform aneurysm: steady flows
}

Shyam Sunder Gopalakrishnan, Benoît Pier, Arie Biesheuvel

\section{To cite this version:}

Shyam Sunder Gopalakrishnan, Benoît Pier, Arie Biesheuvel. Global stability analysis of flow through a fusiform aneurysm: steady flows. Journal of Fluid Mechanics, 2014, 752, pp.90 - 106. 10.1017/jfm.2014.292 . hal-01084523

\section{HAL Id: hal-01084523 https://hal.science/hal-01084523}

Submitted on 19 Nov 2014

HAL is a multi-disciplinary open access archive for the deposit and dissemination of scientific research documents, whether they are published or not. The documents may come from teaching and research institutions in France or abroad, or from public or private research centers.
L'archive ouverte pluridisciplinaire HAL, est destinée au dépôt et à la diffusion de documents scientifiques de niveau recherche, publiés ou non, émanant des établissements d'enseignement et de recherche français ou étrangers, des laboratoires publics ou privés. 


\title{
Global stability analysis of flow through a fusiform aneurysm: steady flows
}

\author{
Shyam Sunder Gopalakrishnan†, Benoît Pier and Arie Biesheuvel \\ Laboratoire de Mécanique des Fluides et d'Acoustique, CNRS - École Centrale de Lyon, \\ Université Claude-Bernard Lyon 1, INSA de Lyon, 36 Avenue Guy de Collongue, 69134 Écully, France
}

(Received 11 July 2013; revised 12 May 2014; accepted 21 May 2014)

The global linear stability of steady axisymmetric flow through a model fusiform aneurysm is studied numerically. The aneurysm is modelled as a Gaussian-shaped inflation on a vessel of circular cross-section. The fluid is assumed to be Newtonian, and the flow far upstream and downstream of the inflation is a Hagen-Poiseuille flow. The model aneurysm is characterized by a maximum height $H$ and width $W$, non-dimensionalized by the upstream vessel diameter, and the steady flow is characterized by the Reynolds number of the upstream flow. The base flow through the model aneurysms is determined for non-dimensional heights and widths in the physiologically relevant ranges $0.1 \leqslant H \leqslant 1.0$ and $0.25 \leqslant W \leqslant 2.0$, and for Reynolds numbers up to 7000 , corresponding to peak values recorded during pulsatile flows under physiological conditions. It is found that the base flow consists of a core of relatively fast-moving fluid, surrounded by a slowly recirculating fluid that fills the inflation; for larger values of the ratio $H / W$, a secondary recirculation region is observed. The wall shear stress (WSS) in the inflation is vanishingly small compared to the WSS in the straight vessels. The global linear stability of the base flows is analysed by determining the eigenfrequencies of a modal representation of small-amplitude perturbations and by looking at the energy transfer between the base flow and the perturbations. Relatively shallow aneurysms (of relatively large width) become unstable by the lift-up mechanism and have a perturbation flow which is characterized by stationary, growing modes. More localized aneurysms (with relatively small width) become unstable at larger Reynolds numbers, presumably by an elliptic instability mechanism; in this case the perturbation flow is characterized by oscillatory modes.

Key words: biological fluid dynamics, instability

\section{Introduction}

Following the observation of transition to turbulence in the human aorta by Nerem, Seed \& Wood (1972), the stability of blood flow in the arterial system has been studied extensively. Shortis \& Hall (1999), Boiron, Deplano \& Pelissier (2007) and Trip et al. (2012), among others, have analysed the stability of pulsatile flow in straight and curved tubes of circular cross-section under physiological conditions.

$†$ Email address for correspondence: shyam-sunder.gopalakrishnan@univ-lyon1.fr 
Recently, because of its association with atherosclerosis, the stability of flow through arterial blockages (stenoses) has been the subject of much research (Sherwin \& Blackburn 2005; Blackburn \& Sherwin 2007; Peterson \& Plesniak 2008; Blackburn, Sherwin \& Barkley 2008; Griffith et al. 2009; Mao, Sherwin \& Blackburn 2011). The structure and stability of flow through arterial inflations (fusiform aneurysms, or abdominal arterial aneurysms), on the other hand, has attracted less attention. Some notable exceptions are the papers by Finol \& Amon (2001), Yip \& Yu (2001), Finol \& Amon (2002b), Salsac et al. (2006) and Sheard (2009); other work is discussed in the reviews by Lasheras (2007) and Humphrey \& Taylor (2008).

Faced with the complexity of analysing pulsatile flows in arterial blockages or inflations, it is sensible to first consider the stability of steady flows in such geometries. Obviously, the results are not physiologically realistic, although they may be relevant for clinical practice and will certainly be helpful in setting up experiments. Thus, the stability of steady flow through arterial stenoses has been addressed by Sherwin \& Blackburn (2005), Griffith et al. (2007), Blackburn et al. (2008), Griffith et al. (2008), Vétel et al. (2008) and Griffith et al. (2013). This paper concentrates on the stability of steady flow through fusiform aneurysms. The velocity field and wall shear stress (WSS) distribution in steady flow through fusiform aneurysms have been studied earlier by, for example, Peattie et al. (1994), Bluestein et al. (1996), Peattie et al. (1996) and Finol \& Amon (2002a).

The model aneurysms studied here are axisymmetric with an inflation of the wall that takes a Gaussian shape. This shape has an advantage over the sinusoidal shapes used in other studies (Sheard 2009) in that its spatial derivatives are continuous to all orders - this is a desirable feature, particularly in the context of studies of hydrodynamic stability; moreover, it is what one would expect to be the case in real aneurysms.

The fluid mechanical problem and the methods of analysis are introduced in $\S 2$, where we also describe the numerical procedures and verification of the code. The results are presented in $\S 3$, i.e. the structure of the steady basic flows through the model aneurysms, the conditions for linear global stability and the structure of the critical amplitude functions. Conclusions are given in $\S 4$.

\section{Methods}

\subsection{Problem formulation}

The model aneurysm considered in this paper is shown schematically in figure 1. Let $r$ denote the distance from the centreline of the vessel and $z$ the distance along the centreline, with the origin placed at the axial midpoint of the inflation; then the vessel wall $r=R(z)$ is defined by

$$
R(z)=\frac{1}{2} D+H \exp \left(-\frac{1}{2} z^{2} / W^{2}\right) .
$$

Here $D$ is the vessel diameter far upstream and downstream of the inflation, $H$ is the maximum height of the inflation and $W$ is a measure of the width of the inflation. For comparison, the vessel with a sinusoidally shaped inflation studied by Sheard (2009), or Model 3 in Salsac et al. (2006), is indicated in figure 1 by the dotted lines. It is important to note that the parameter $W$ is a measure of the length of the abdominal arterial aneurysm, and is not the wavelength of the sinusoidal function used to model the aneurysm dilatation in Sheard (2009). To cover different stages of development of an aneurysm, the following parameter ranges are considered: $0.25 \leqslant W / D \leqslant 2$ and 


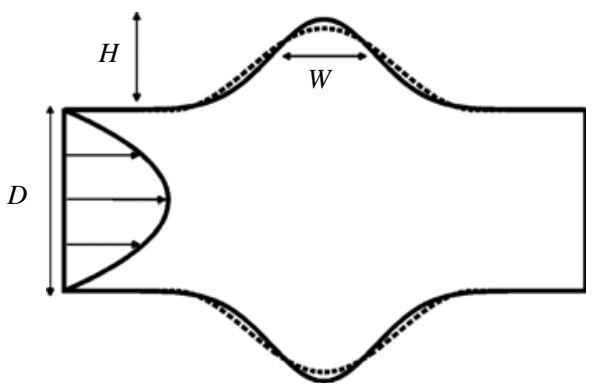

FIGURE 1. The cylindrical geometry considered in the present study, with the radius described by a Gaussian function. In this example $W / D=0.5$ and $H / D=0.5$. The sinusoidal shape used in Sheard (2009) and Model 3 of Salsac et al. (2006) is shown by the dotted lines.

$0.1 \leqslant H / D \leqslant 1$. The latter corresponds to a dilatation ratio of $1.2 \leqslant D_{R} \leqslant 3$, where $D_{R}=1+2 H / D$ is the ratio of the maximum diameter of the inflation to the diameter of the non-inflated vessel.

The fluid motion is assumed to be incompressible and governed by the NavierStokes equations. The no-slip condition prevails at the vessel wall, which is assumed to be rigid. The fluid velocity distribution is taken to converge to that of a Hagen-Poiseuille flow far upstream and downstream of the inflation. The Reynolds number of the flow is defined as $R e=\bar{U} D / v$, where $v$ is the kinematic viscosity of the fluid and $\bar{U}$ is the (specified) cross-sectionally averaged velocity in the vessel. Reynolds numbers up to $R e=7000$ are considered, in view of the fact that typical peak Reynolds numbers of pulsatile blood flow in the abdominal region of the aorta

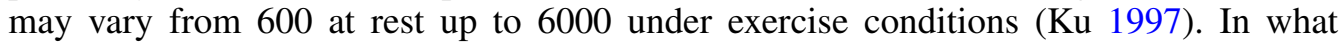
follows, all lengths and velocities are non-dimensionalized by the vessel diameter $D$ and the mean velocity $\bar{U}$, respectively.

\subsection{Stability analysis}

Using cylindrical coordinates, the radial velocity $U_{r}(r, z)$, the axial velocity $U_{z}(r, z)$ and the pressure $P(r, z)$ of the steady axisymmetric flow through the aneurysms are governed by

$$
\begin{gathered}
U_{r} \frac{\partial U_{r}}{\partial r}+U_{z} \frac{\partial U_{r}}{\partial z}=-\frac{\partial P}{\partial r}+\frac{1}{R e}\left[\frac{1}{r} \frac{\partial}{\partial r}\left(r \frac{\partial U_{r}}{\partial r}\right)+\frac{\partial^{2} U_{r}}{\partial z^{2}}-\frac{U_{r}}{r^{2}}\right], \\
U_{r} \frac{\partial U_{z}}{\partial r}+U_{z} \frac{\partial U_{z}}{\partial z}=-\frac{\partial P}{\partial z}+\frac{1}{R e}\left[\frac{1}{r} \frac{\partial}{\partial r}\left(r \frac{\partial U_{z}}{\partial r}\right)+\frac{\partial^{2} U_{z}}{\partial z^{2}}\right], \\
\frac{\partial U_{r}}{\partial r}+\frac{U_{r}}{r}+\frac{\partial U_{z}}{\partial z}=0
\end{gathered}
$$

with the no-slip condition $U_{r}=U_{z}=0$ along the wall

$$
r=R(z) \equiv \frac{1}{2}+H \exp \left(-\frac{1}{2} z^{2} / W^{2}\right)
$$

and with the following conditions far upstream and downstream $(z \rightarrow \pm \infty)$ :

$$
U_{r}=0, \quad U_{z}=2\left(1-4 r^{2}\right) .
$$


The linear stability of the base flow $(\boldsymbol{U}, P)$ is investigated by superimposing a three-dimensional small-amplitude perturbation $\left(\boldsymbol{u}^{\prime}, p^{\prime}\right)$. The linearized Navier-Stokes equations governing the behaviour of the perturbation are

$$
\begin{aligned}
\frac{\partial \boldsymbol{u}^{\prime}}{\partial t}+(\boldsymbol{U} \cdot \nabla) \boldsymbol{u}^{\prime}+\left(\boldsymbol{u}^{\prime} \cdot \nabla\right) \boldsymbol{U} & =-\nabla p^{\prime}+\frac{1}{R e} \Delta \boldsymbol{u}^{\prime}, \\
\nabla \cdot \boldsymbol{u}^{\prime} & =0 .
\end{aligned}
$$

The steadiness and axisymmetry of the base flows allow us to write the perturbations in the modal form

$$
\left[\boldsymbol{u}^{\prime}, p^{\prime}\right](r, \theta, z, t)=\left[\hat{u}_{r}, \hat{u}_{\theta}, \hat{u}_{z}, \hat{p}\right](r, z) \mathrm{e}^{\mathrm{i}(m \theta-\omega t)},
$$

where $m$ is the (integer) azimuthal wavenumber and $\omega \equiv \omega_{r}+\mathrm{i} \omega_{i}$ is the complex eigenfrequency. The perturbation is stationary when $\omega_{r}=0$ and oscillatory when $\omega_{r} \neq$ 0 ; when the growth rate $\omega_{i}$ is positive, the perturbation grows exponentially in time.

Substitution of (2.6) into the linearized Navier-Stokes equations (2.5) yields a generalized eigenvalue problem of the form

$$
\boldsymbol{A} \hat{\boldsymbol{u}}=-\mathrm{i} \omega \boldsymbol{B} \hat{\boldsymbol{u}}
$$

where $\hat{\boldsymbol{u}}=\left(\hat{u}_{r}, \hat{u}_{\theta}, \hat{u}_{z}, \hat{p}\right)$ is a vector consisting of the amplitude functions and $\boldsymbol{A}$ and $\boldsymbol{B}$ are the matrices associated with (2.5). A discussion of methods of global linear instability analysis, together with many examples, can be found in Theofilis (2011).

\subsection{Energy transfer analysis}

The rate of change of the kinetic energy of the perturbation flow is governed by the Reynolds-Orr energy equation

$$
\frac{\mathrm{d}}{\mathrm{d} t}\left(\int_{V} \frac{1}{2} u_{i}^{\prime} u_{i}^{\prime} \mathrm{d} V\right)=-\frac{1}{R e} \int_{V}\left(\frac{\partial{u_{i}^{\prime}}^{2}}{\partial x_{j}}\right) \mathrm{d} V-\int_{V} \frac{1}{2} u_{i}^{\prime} u_{j}^{\prime}\left(\frac{\partial U_{i}}{\partial x_{j}}+\frac{\partial U_{j}}{\partial x_{j}}\right) \mathrm{d} V,
$$

where contributions due to advection of kinetic energy through the domain boundaries have been neglected. The first term on the right-hand side of (2.8) represents the viscous dissipation of kinetic energy, and the second term represents the transfer of kinetic energy from the base flow to the perturbations. This equation has proven to be helpful in deciding which instability mechanisms are involved in the evolution of the perturbations.

Following Lanzerstorfer \& Kuhlmann (2012), the perturbation velocity $\boldsymbol{u}^{\prime}$ is decomposed into components $\boldsymbol{u}_{\|}^{\prime}$ and $\boldsymbol{u}_{\perp}^{\prime}$ which are, respectively, parallel and perpendicular to the base velocity $\boldsymbol{U}$ :

$$
\boldsymbol{u}_{\|}^{\prime}=\frac{\left(\boldsymbol{u}^{\prime} \cdot \boldsymbol{U}\right) \boldsymbol{U}}{\boldsymbol{U} \cdot \boldsymbol{U}}, \quad \boldsymbol{u}_{\perp}^{\prime}=\boldsymbol{u}^{\prime}-\boldsymbol{u}_{\|}^{\prime},
$$

such that the local energy transfer from the base flow to the perturbations may be written as

$$
\sum_{i=1}^{4} E_{p, i}=-\left[\boldsymbol{u}_{\perp}^{\prime} \cdot\left(\boldsymbol{u}_{\perp}^{\prime} \cdot \nabla \boldsymbol{U}\right)+\boldsymbol{u}_{\|}^{\prime} \cdot\left(\boldsymbol{u}_{\perp}^{\prime} \cdot \nabla \boldsymbol{U}\right)+\boldsymbol{u}_{\perp}^{\prime} \cdot\left(\boldsymbol{u}_{\|}^{\prime} \cdot \nabla \boldsymbol{U}\right)+\boldsymbol{u}_{\|}^{\prime} \cdot\left(\boldsymbol{u}_{\|}^{\prime} \cdot \nabla \boldsymbol{U}\right)\right]
$$


Here, the index $i$ numbers all terms on the right-hand side consecutively. The use of coordinates which are locally aligned with the flow direction is appropriate because locally no other distinguished direction exists. Each of the terms $E_{p, i}$ may be viewed as representing a different energy transfer process. For example, $E_{p, 2}$ represents the local rate of change of the energy density of the perturbations due to convective transport by the cross-stream perturbation velocity component $\boldsymbol{u}_{\perp}^{\prime}$ of the basic-state momentum per unit mass, $\boldsymbol{U}$. Hence, if $\boldsymbol{u}_{\perp}^{\prime} \cdot \boldsymbol{\nabla} \boldsymbol{U}$ has a component parallel to and in the direction of $\boldsymbol{u}_{\|}^{\prime}$, kinetic energy is locally transferred from the base flow to the perturbations. When $E_{p, 2}<0$, the perturbations locally lose energy to the base flow.

\subsection{Numerical methods}

The flow problem (2.2)-(2.4) and the generalized eigenvalue problem (2.5)-(2.7) are solved numerically by a finite element method. The spatial discretization is a mixed finite element formulation using P2 and P1 Taylor-Hood elements, i.e. six-node quadratic triangular elements with quadratic interpolation for velocities (P2) and three-node linear triangular elements for pressure (P1). The meshes and the discrete matrices resulting from the variational formulation of the problems are generated with the software FreeFem++ (http://www.freefem.org).

The base flows are computed as in Marquet et al. (2009). First, a steady solution of the full time-dependent Navier-Stokes equations is computed, for a small Reynolds number such that the flow is stable. Next, a Newton-Raphson iteration method is used to compute a solution of the steady Navier-Stokes equations, starting with the small-Reynolds-number solution as a first guess, and approaching the required largeReynolds-number solution by incrementing the Reynolds number in small steps.

Once a base flow has been computed, the associated linear global stability problem is solved. To compute the eigenfrequencies with largest imaginary part, first a shiftand-invert strategy is used, as in Ehrenstein \& Gallaire (2005) and Marquet et al. (2009), to single out the region of interest in the complex frequency plane. The result is an eigenvalue problem, which is then solved with the implicitly restarted Arnoldi algorithm provided by the UMFPACK software library. The method is discussed in detail in Sorensen (1992) and Nayar \& Ortega (1993).

\subsection{Code validation}

To validate the computation of the base flows, grid convergence and independence of the size of the computational domain were addressed. Table 1 gives information on the meshes $M$ used for the vessel geometry with $W=0.5$ and $H=0.5$, representative of the tests that were carried out. The size of the computational domain is characterized by $\Omega$, which gives the axial extension measured in vessel diameters with respect to the origin. The number of nodes associated with the P2 and P1 elements of a mesh are denoted by $N_{2}$ and $N_{1}$, respectively. The subscript $b$ indicates that the mesh is associated with the computation of a base flow; the subscripts $s$ and $l$ indicate that the computational domain is, respectively, smaller or larger than the 'standard domain' $(-10,10)$.

To check for convergence when computing the base flow fields, the WSS is used as a sensitive measure of the overall grid resolution. The WSS distribution follows from evaluation of the expression

$$
2\left(\frac{\partial U_{r}}{\partial r}-\frac{\partial U_{z}}{\partial z}\right) \cos \alpha \sin \alpha-\left(\frac{\partial U_{r}}{\partial z}+\frac{\partial U_{z}}{\partial r}\right)\left[\cos ^{2} \alpha-\sin ^{2} \alpha\right]
$$




\begin{tabular}{lcrr} 
Mesh & \multicolumn{1}{c}{$\Omega$} & \multicolumn{1}{c}{$N_{2}$} & \multicolumn{1}{c}{$N_{1}$} \\
$M_{b}$ & $(-10,10)$ & 183061 & 46516 \\
$M_{b 1}$ & $(-10,10)$ & 152449 & 38788 \\
$M_{b 2}$ & $(-10,10)$ & 66398 & 17050 \\
$M_{b 3}$ & $(-10,10)$ & 17058 & 4490 \\
$M_{b s}$ & $(-5,5)$ & 136049 & 34613 \\
$M_{b l}$ & $(-20,20)$ & 229558 & 58515
\end{tabular}

TABLE 1. Meshes used to validate the computation of the base flow for $H=0.5$ and $W=0.5$.

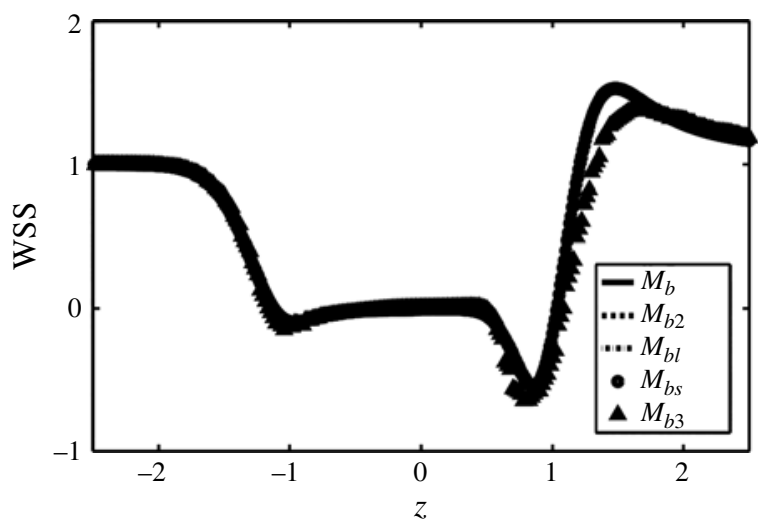

FIGURE 2. The distribution of WSS computed using the different meshes listed in table 1. The parameter settings are $H=0.5, W=0.5$ and $R e=2500$.

at the vessel wall $r=R(z)$. In this expression $\alpha(z)$ denotes the local angle of wall slope, defined by $\tan \alpha=\mathrm{d} R / \mathrm{d} z$. A negative value of the WSS corresponds to reversed flow.

Figure 2 presents the results of the computation of the WSS distribution in a vessel inflation using the meshes listed in table 1. This distribution is normalized by the value of the WSS exerted by the associated Hagen-Poiseuille flow. The Reynolds number is 2500. It is observed that where the vessel is inflated, corresponding roughly to the range $-1<z<1$, the WSS is reduced significantly; in fact, it becomes vanishingly small. Near the downstream end of the inflation, however, a sudden change occurs, from a negative relative value of one-half to a positive relative value of one-and-a-half. Similar observations on WSS distributions were made by Budwig et al. (1993) and Bluestein et al. (1996). With the exception of the computations done with the rather coarse mesh $M_{b 3}$, all the computations match perfectly with those carried out on the fine mesh $M_{b l}$.

Next, we consider the validation of the stability calculations. As before, the results of tests carried out for the values $W=0.5, H=0.5$ and $R e=2500$ are presented. The meshes used in this case are specified in table 2 , which also reports the computed values of the real and imaginary parts of $\omega_{1}$, the eigenfrequency with the largest imaginary part associated with wavenumber $m=1$. With the exception of the coarse mesh $M_{s 3}$, all meshes yield values for the eigenfrequencies which agree up to the fourth digit. This finding permits us to use mesh $M_{s 2}$ for the stability computations. 


\begin{tabular}{lcrrcc} 
Mesh & \multicolumn{1}{c}{$\Omega$} & \multicolumn{1}{c}{$N_{2}$} & \multicolumn{1}{c}{$N_{1}$} & \multicolumn{1}{c}{$\omega_{1 i}$} & $\omega_{1 r}$ \\
$M_{s 1}$ & $(-10,10)$ & 124566 & 31742 & -0.0301923 & 0.0310782 \\
$M_{s 2}$ & $(-10,10)$ & 66398 & 17050 & -0.0301750 & 0.0311178 \\
$M_{s 3}$ & $(-10,10)$ & 17058 & 4490 & -0.0293571 & 0.0304471 \\
$M_{s 0}$ & $(-10,10)$ & 152449 & 38788 & -0.0302183 & 0.0310903 \\
$M_{b s}$ & $(-5,5)$ & 136049 & 34613 & -0.0302199 & 0.0310871
\end{tabular}

TABLE 2. Size of the computational domain, as characterized by $\Omega$, and the number of nodes $N_{1}$ and $N_{2}$ used in convergence tests of the stability analysis; $\omega_{1 i}$ and $\omega_{1 r}$ are the computed imaginary and real parts of the eigenfrequency of the wavenumber $m=1$. Here the parameter values are $H=0.5, W=0.5$ and $R e=2500$.

\begin{tabular}{rcrcrrr}
\multicolumn{7}{c}{$(a)$} \\
\hline$L_{o}$ & $b$ & \multicolumn{1}{c}{$\operatorname{Re}$} & $m$ & \multicolumn{1}{c}{$N_{2}$} & \multicolumn{1}{c}{$N_{1}$} & \multicolumn{1}{c}{$\omega_{1 i}$} \\
40 & 0.500 & 2000 & 2 & 114578 & 29470 & -0.01490938 \\
20 & 0.500 & 2350 & 2 & 28310 & 7478 & 0.00025731 \\
20 & 0.750 & 770 & 1 & 8258 & 31230 & -0.00014151
\end{tabular}

\begin{tabular}{rcrcc}
\multicolumn{5}{c}{$(b)$} \\
\hline$L_{o}$ & $b$ & \multicolumn{1}{c}{$\operatorname{Re}$} & $m$ & $\omega_{1 i}$ \\
40 & 0.500 & 2000 & 2 & -0.014783 \\
40 & 0.500 & 2350 & 2 & 0.00000 \\
40 & 0.750 & 770 & 1 & 0.00000
\end{tabular}

TABLE 3. (a) Results of the global stability calculations of steady flow through a stenosis; $R e$ is based on the inlet diameter $D$ and the cross-sectionally averaged velocity $\bar{U}$, and the growth rate $\omega_{1 i}$ has been normalized by $\bar{U} / D$. (b) Corresponding results from Griffith et al. (2008).

Extensive validation tests have been performed at higher Reynolds numbers and for a range of bulge heights, which are discussed in detail in Gopalakrishnan (2014).

We have also performed computations of flow through model arterial stenoses, to verify our numerical results against those of Griffith et al. (2008). Flow through a stenosis presents different numerical challenges than flow through an aneurysm. Large regions of recirculating flow occur downstream of a stenosis, which makes the proper choice of the length of the straight vessel outlet an important issue. In flow through an aneurysm, the recirculating flow, as well as the extent of the critical amplitude functions, is limited to the region of vessel inflation. Table 3 presents a comparison between our results and some of those from Griffith et al. (2008). Here the parameter $b$ is the stenosis degree, defined as

$$
b=1-\left(\frac{d}{D}\right)^{2}
$$

where $d$ is the diameter of the vessel at the centre of the blockage and $L_{0}$ denotes the length of the vessel outlet in units of vessel diameter.

\section{Results}

\subsection{Base flows}

Streamlines and distributions of azimuthal vorticity of the steady axisymmetric base flows through inflated vessels are shown in figures 3-5. In these figures the values of the dimensionless width $W$ and Reynolds number $\operatorname{Re}$ are: $W=2.0$ and $R e=4000$ for figure 3; $W=0.5$ and $R e=5500$ for figure 4 ; and $W=0.25$ and $R e=5500$ for 

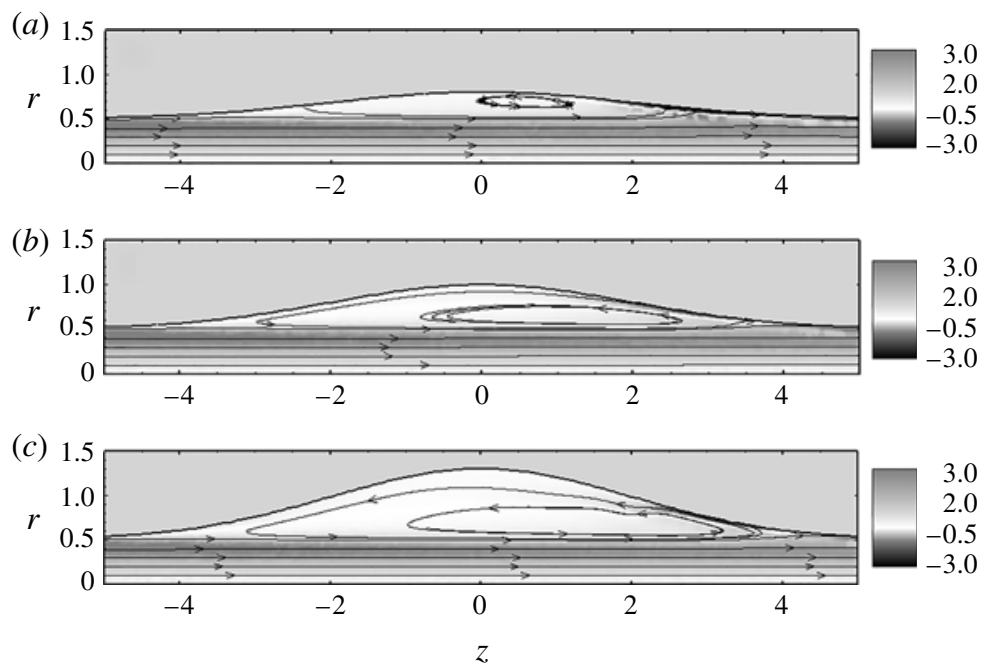

FIGURE 3. Basic flow visualized by the vorticity and streamlines at $R e=4000$ for $W=2.0$. The parameter settings are: $(a) H=0.3 ;(b) H=0.5$; (c) $H=0.8$.
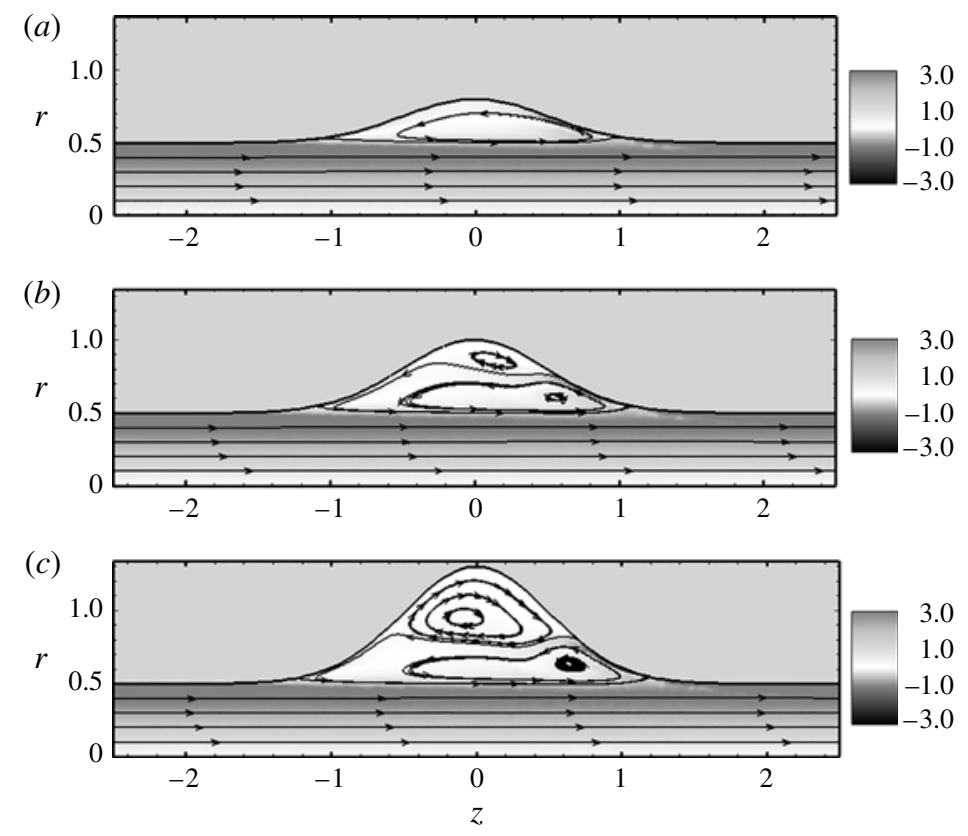

FIGURE 4. As figure 3, but with $R e=5500$ and $W=0.5$.

figure 5. In each of these figures the dimensionless height is $H=0.3$ in $(a), H=0.5$ in $(b)$ and $H=0.8$ in $(c)$. Note that, to bring out details, the scales in the figures have been chosen differently. We would like to remind the reader that the peak Reynolds numbers in the abdominal aorta can vary from 600 at rest up to 6000 under exercise conditions ( $\mathrm{Ku}$ 1997).

The flow through the model aneurysms is qualitatively similar for all parameter values such that $H / W$ is relatively small, consisting of a jet-like core of diameter 

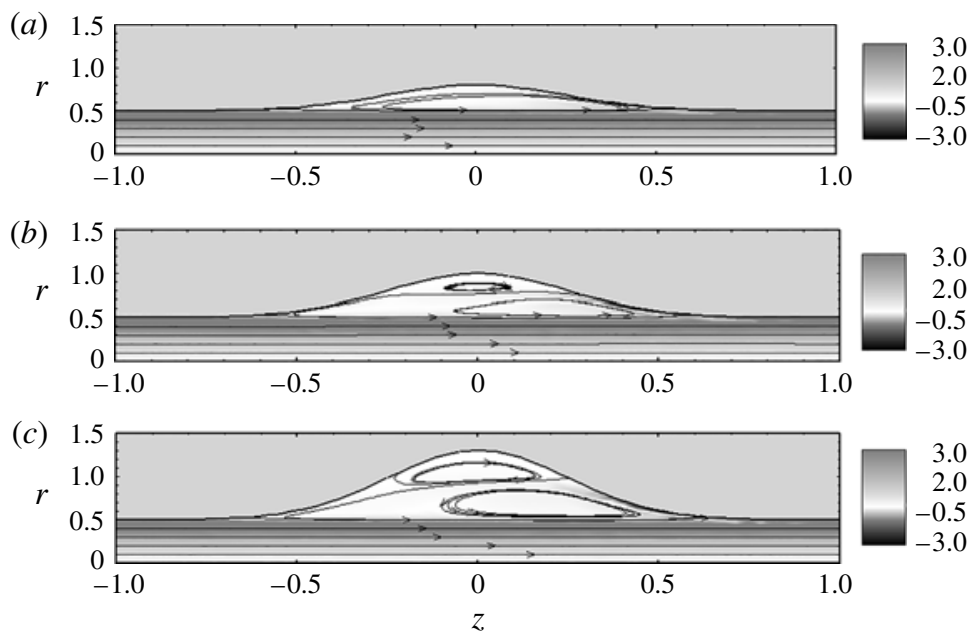

FIgURE 5. As figure 3, but with $R e=5500$ and $W=0.25$.

roughly that of the non-inflated vessel, surrounded by slowly recirculating fluid in the inflation. In the recirculation region, the radial velocity is at least two orders of magnitude smaller than the axial velocity, except near the downstream end of the inflation, where it is approximately one order of magnitude smaller. The vorticity distribution in the core region is uniform, with magnitude similar to that in the Hagen-Poiseuille flow far upstream and downstream. The vorticity distribution in the recirculation region is also approximately uniform, but of opposite sign and smaller magnitude. Near the stagnation point at the downstream end of the inflation, there is a small region in which the vorticity is vanishingly small. This flow structure has been observed by Budwig et al. (1993) and Bluestein et al. (1996) in their experiments and numerical calculations.

The migration with increasing Reynolds number of the centre of the recirculation zone towards the distal end, resulting in a concentration of vorticity at the downstream end, was reported in Bluestein et al. (1996). However, it has not been reported before that a secondary recirculation region appears, with weak vorticity of opposite sign to that in the primary recirculation zone, as the ratio $H / W$ or the Reynolds number $R e$ is increased. The centre of the primary recirculation zone is displaced towards the distal end of the inflation as $H / W$ or $R e$ is increased further, while the centre of the secondary recirculation zone is displaced towards the proximal end of the inflation.

\subsection{Critical modes and stability boundaries}

A typical eigenfrequency spectrum in the complex $\omega$-plane is shown in figure 6 . In this example $W=0.5, H=0.5$ and $R e=5500$, parameter values for which the basic flow is shown in figure $4(b)$. Two modes with a positive temporal growth rate $\omega_{i}$ can be observed; they are associated with the azimuthal wavenumbers $m=4$ and $m=5$. The real parts $\omega_{r}$ of these eigenfrequencies are non-zero, so that the unstable modes represent three-dimensional oscillations which grow in amplitude.

With each azimuthal wavenumber $m$ a 'leading mode' can be associated; this is the mode with the largest imaginary part $\omega_{i}$ of the eigenfrequency. The variation of the temporal growth rate $\omega_{i}$ of these leading modes with Reynolds number is shown in figure $7(a)$, again for $W=0.5$ and $H=0.5$. Figure $7(b)$ shows the real part $\omega_{r}$ of the 

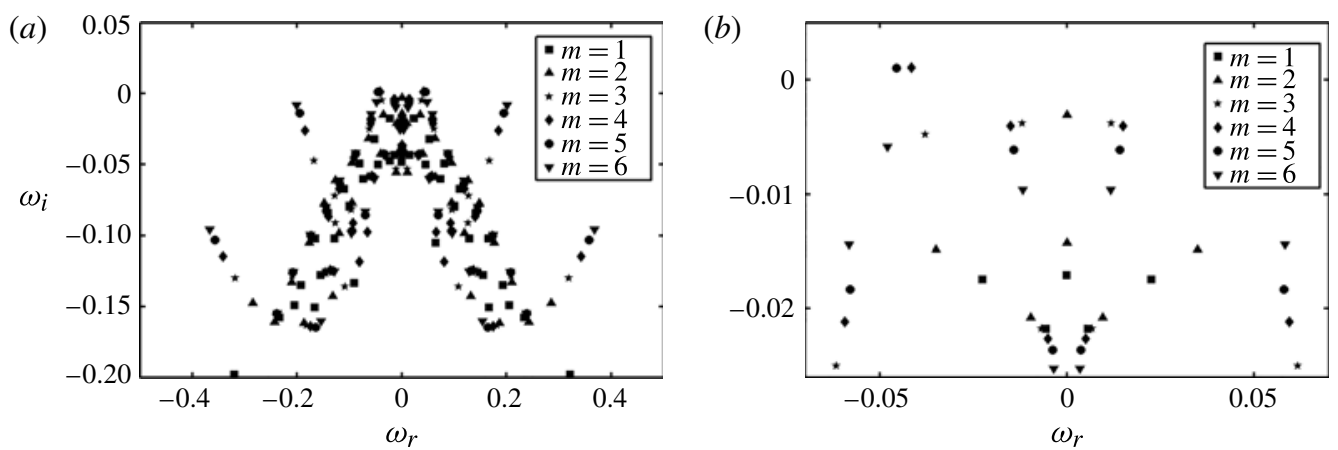

FIGURE 6. (a) Eigenfrequency spectrum for different azimuthal wavenumbers $m$, in the setting where $W=0.5, H=0.5$ and $R e=5500$. (b) A close-up view of the eigenfrequency spectrum, showing the unstable modes corresponding to $m=4$ and $m=5$.
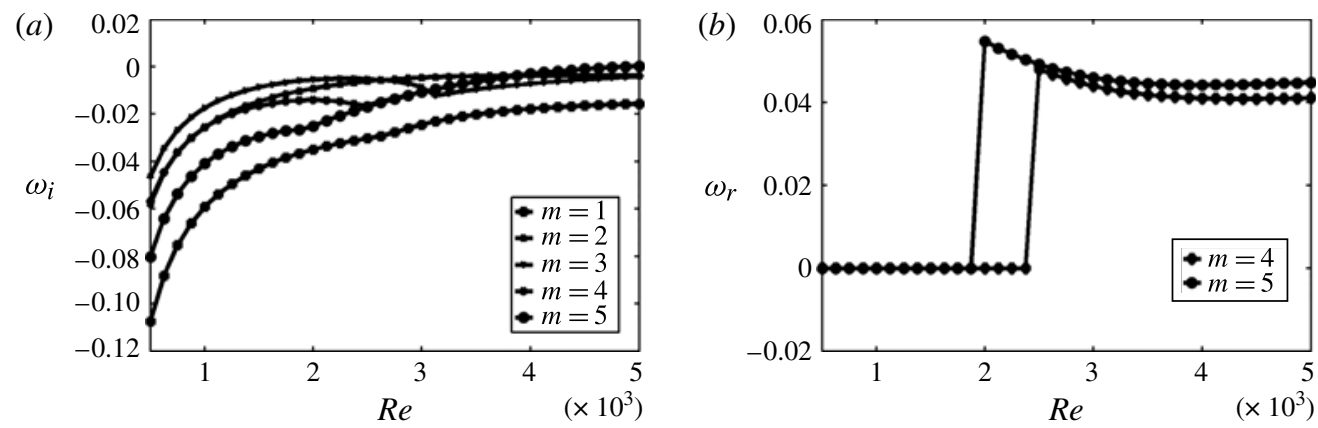

FIGURE 7. Plots as a function of the Reynolds number $R e$ of the leading modes associated with different azimuthal wavenumbers $m$, in the setting where $W=0.5$ and $H=0.5$ : (a) temporal growth rates $\omega_{i} ;(b)$ oscillation frequencies $\omega_{r}$.

leading modes associated with wavenumbers $m=4$ and $m=5$. The temporal growth rates of both of these modes become positive at $R e \approx 4875$; both modes are found to switch abruptly from stationary to oscillatory at much smaller Reynolds numbers.

The stability boundaries in the $R e-H$ plane for different azimuthal wavenumbers $m$ are shown in figure 8, for four values of the bulge width: (a) $W=2.0 ;(b) W=1.0$; (c) $W=0.5 ;(d) W=0.25$. For small values of the height $H$, the aneurysm can be viewed as a small perturbation to a fully developed flow in a circular pipe; that flow is linearly stable for all Reynolds numbers. Although a critical value cannot be given, it is fair to say that the base flow is linearly stable for bulge heights $H<$ 0.2 (for Reynolds numbers $R e<7000$ ). As the height $H$ is increased, the base flow becomes unstable to stationary critical modes; these are indicated by the dotted curves in figure 8. The azimuthal wavenumbers that correspond to these critical modes differ according to the width $W$. No trend is observed, although the wavenumbers $m=2$ and $m=5$ seem to be involved most often. As the bulge height is increased further, the base flow also becomes unstable to oscillatory modes, as indicated by the solid curves in figure 8 . With regard to the influence of the width of the inflation, we find that as the width is reduced from $W=2.0$ to $W=0.5$, the base flow becomes unstable at smaller values of the Reynolds number and of the height of the inflation. 

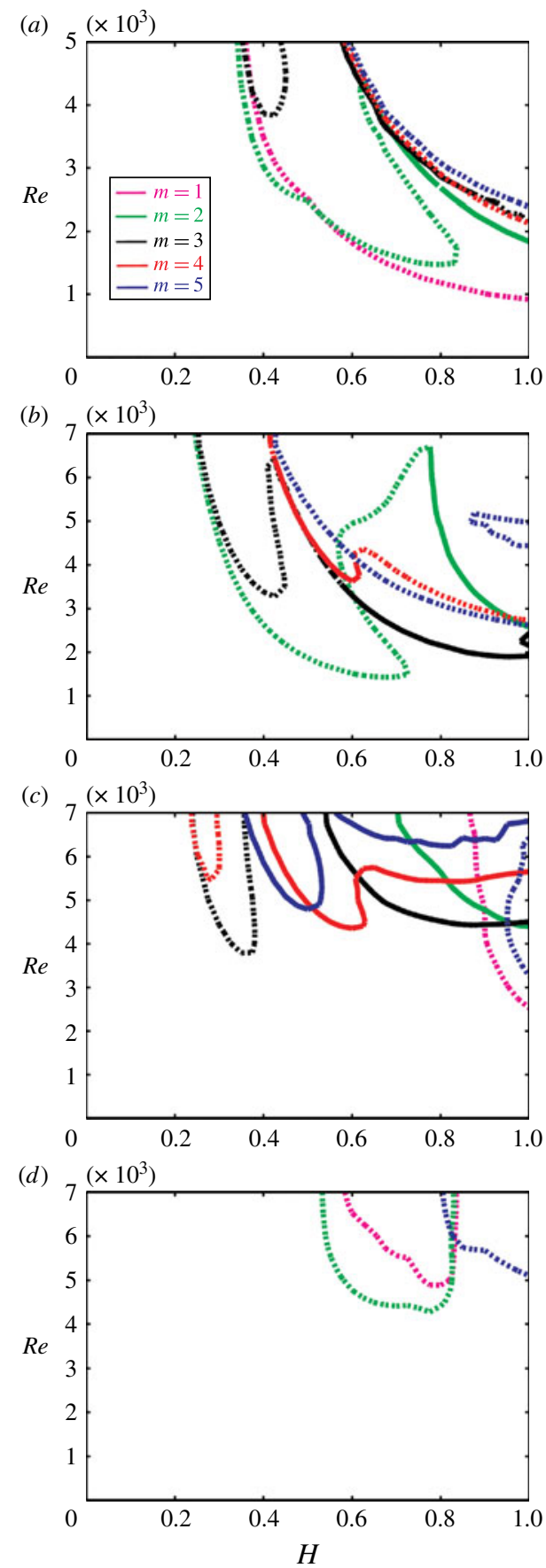

FIGURE 8. (Colour online) Critical curves associated with different azimuthal wavenumbers $m$ in the $R e-H$ plane, where the width of the inflation is: $(a) W=2.0$; (b) $W=1.0 ;(c) W=0.5 ;(d) W=0.25$. The solid curves indicate oscillatory modes and the dotted curves stationary modes. 
(a)

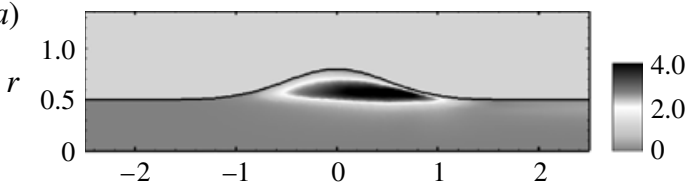

(c)

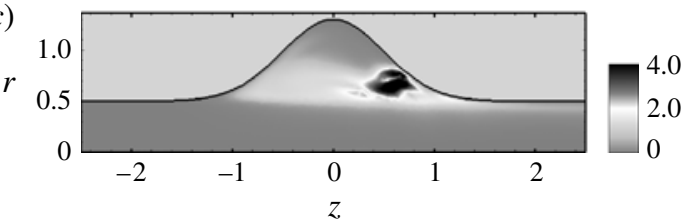

(b)

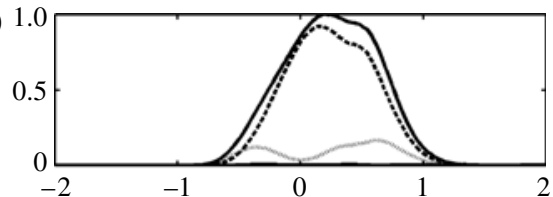

(d)

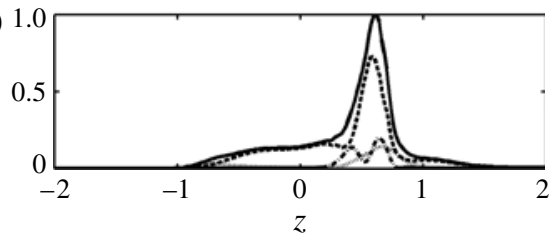

FIGURE 9. (a,c) Amplitude of the global mode defined as the square root of the perturbation energy density field. $(b, d)$ Variation with axial distance $z$ of the magnitude of the velocity of the global mode and of its components integrated over $r$, all normalized by the maximum value of $\int|\hat{\boldsymbol{u}}|^{2} \mathrm{~d} r: \int|\hat{\boldsymbol{u}}|^{2} \mathrm{~d} r$ (solid); $\int\left|\hat{u}_{z}\right|^{2} \mathrm{~d} r$ (dashed); $\int\left|\hat{u}_{r}\right|^{2} \mathrm{~d} r$ (dot-dash); $\left.\int\left|\hat{u_{\theta}}\right|^{2} \mathrm{~d} r\right)$ (dotted). The parameter values are $R e=5500, m=3, W=0.5$ and $(a, b) H=0.3$; $(c, d) H=0.8$.

However, a further reduction of the width improves the linear stability of the base flows significantly.

\subsection{Instability mechanisms}

The analysis of the energy transfer processes that take place suggest that different instability mechanisms are involved when the base flow has just a single recirculation region versus when it also includes a secondary recirculation region. To see this, one can compare two flows at $R e=5500$ through a model aneurysm of width $W=0.5$, where in one case the bulge height is $H=0.3$ and in the other case it is $H=0.8$. From figure $8(c)$ it can be seen that a stationary mode with wavenumber $m=3$ is unstable for $H=0.3$, whereas for $H=0.8$ this mode is oscillatory.

Figure 9 presents the perturbation energy density distribution associated with the amplitude function $\left(\hat{u}_{r}, \hat{u}_{\theta}, \hat{u}_{z}\right)$ for two different cases: $(a, b)$ the basic flow with a single recirculation region $(H=0.3) ;(c, d)$ the basic flow with also a secondary recirculation region. We observe (see figure $9 a, c$ ) that in the first case the disturbance energy is distributed all along the axis of the inflation, while in the second case it is localized in a small region at the downstream end. This can be seen even more clearly from the cross-sectional averages of the energy density associated with the individual perturbation velocity components (figure $9 b, d$ ). Note also that in both cases, most of the perturbation energy is in the axial perturbation velocity component, and that in the second case there is also a small contribution of the radial velocity component, which is totally absent in the first case; in the first case, then, the disturbance motion takes place in circular planes.

That different mechanisms are involved in the instability of the two basic flows is also clear from figures 10 and 11, which provide information on the energy transfer between the basic flow and the perturbation flow. In each figure, the total energy transfer rate is shown in panel $(d)$, and the three separate contributions $E_{p, 1}, E_{p, 2}$ and $E_{p, 4}$, which were defined in (2.10), are shown in panels $(a),(b)$ and $(c)$, respectively. The global picture is the same as that which emerges from figure 9, but here we can further see that the main energy transfer mechanism in both cases is the one 
(a)

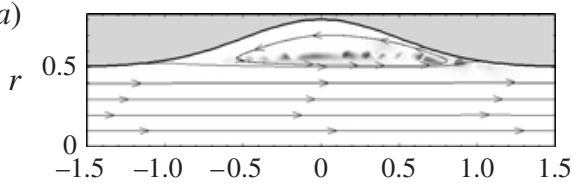

(c)

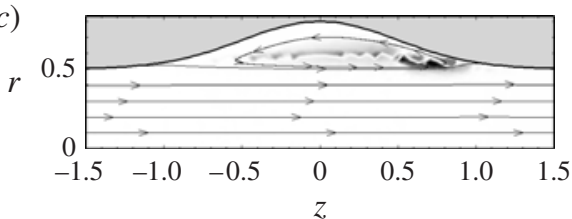

(b)

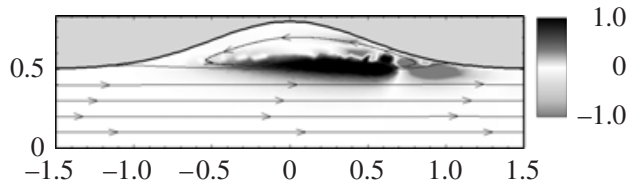

(d)

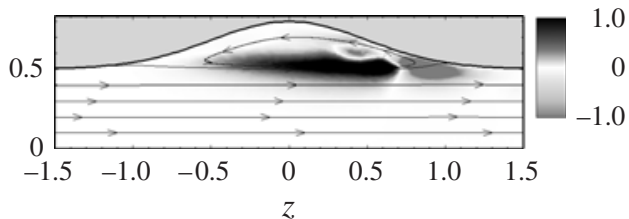

FIGURE 10. Basic streamlines and local energy production rates for $H=0.3$ at $R e=5500$ and for an azimuthal mode number $m=3$ : (a) $E_{p, 1}$; (b) $E_{p, 2} ;$ (c) $E_{p, 4} ;$ (d) $\sum_{i=1}^{4} E_{p, i}$.

(a)

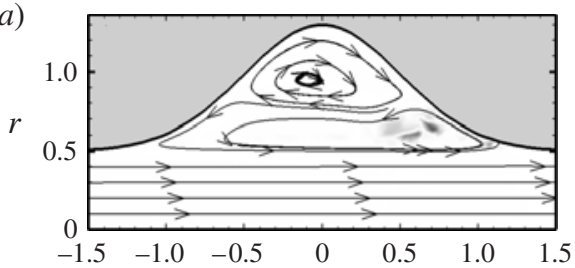

(c)



(b)

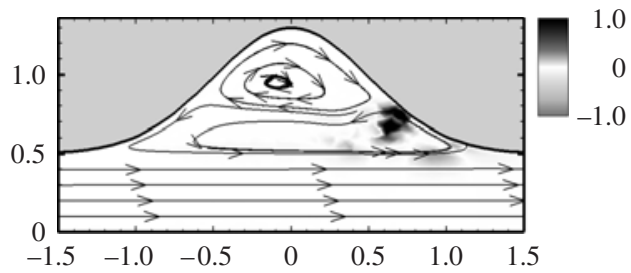

$(d)$

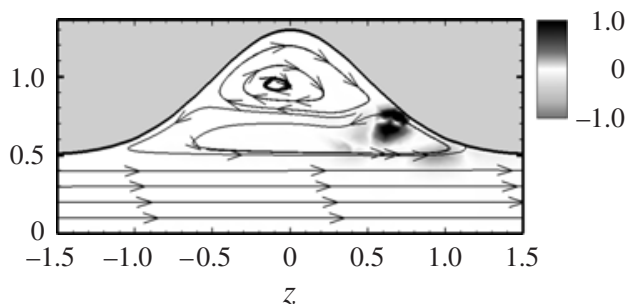

FIGURE 11. Basic streamlines and local energy production rates for $H=0.8$ at $R e=5500$ and for an azimuthal mode number $m=3$ : (a) $E_{p, 1}$; (b) $E_{p, 2} ;(c) E_{p, 4} ;$ (d) $\sum_{i=1}^{4} E_{p, i}$.

represented by $E_{p, 2}$; the locations at which this mechanism is active are different, though.

What are the instability mechanisms? Although this cannot be established rigorously, we consider two typical cases, corresponding to a shallow cavity with $H=0.3$ and a deeper cavity with $H=0.8$, for which our computations suggest the following: in the case with a relatively shallow inflation $(H=0.3)$, the unstable mode is stationary and the disturbance motion primarily takes place on cylindrical planes. Figures 12 and 13 provide a more detailed view of this perturbation velocity field. The picture that emerges is one of a flow with alternating slow and fast streamwise streaks (figure 12) and an arrangement of alternatingly rotating vortices distributed in the layer between the core and the recirculation zone (figure 13). These vortices transport low-velocity fluid to high-speed regions within the perturbation flow. This is the familiar lift-up mechanism, first described by Orr in 1907. The lift-up mechanism has also been identified by Lanzerstorfer \& Kuhlmann (2012) as being the mechanism 


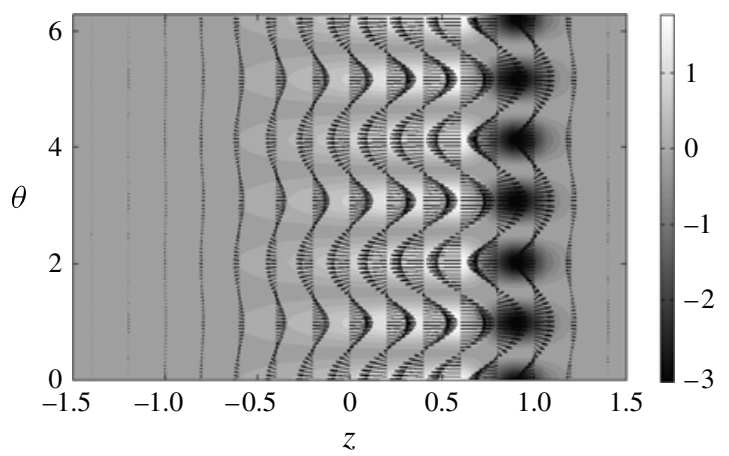

FIGURE 12. Velocity distribution at $r=0.5$ of the most unstable mode associated with wavenumber $m=3$ in the form of streaks (arrows) and the total local energy production $\sum_{i=1}^{4} E_{p, i}$ (grey shading). The parameter values are $H=0.3, W=0.5$ and $R e=5500$.

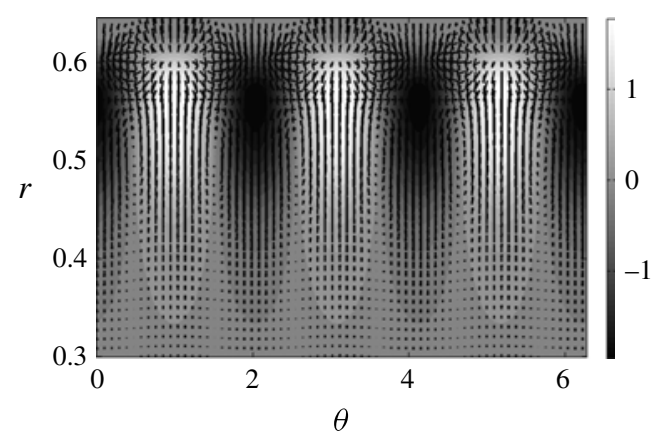

FIGURE 13. Velocity distribution (arrows) and streamwise perturbation velocity $u_{z}^{\prime}$ (grey shading) at the axial location of maximum perturbation energy of the most unstable mode associated with wavenumber $m=3$. The parameter values are $H=0.3, W=0.5$ and $R e=$ 5500 .

that governs the instability of two-dimensional flow past a backward-facing step with small expansion ratio; compare their figure 20 with our figure $10(d)$.

For the more localized inflations (with $H=0.8$ ), it can be seen from figure $11(d)$ that the energy transfer to the perturbation occurs only in a small region, namely at the centre of the primary vortex. This is typical of an elliptical instability mechanism. Lanzerstorfer \& Kuhlmann (2012) have also demonstrated that the elliptical instability mechanism is involved in the global instability of flow past a two-dimensional backward-facing step of moderately large expansion rate; note the close correspondence between our figure $11(d)$ and their figures 13 and 14. This is further exemplified in figure 14(a), where the total local energy production for $H=0.5$ at Reynolds number $R e=5500$ for an azimuthal mode number $m=4$ is shown along with the critical velocity field vectors and the basic flow streamlines. We can see that the perturbation flow is strongest where the energy transfer is maximal, which is entirely localized in the core region of the primary recirculation region, and that they are aligned with the principal direction of strain. Figure 14(b) shows the resulting perturbation flow that arises as a sequence of counter-rotating vortices in the azimuthal plane. The same features are observed at larger bulge heights, which indicates that the instability mechanism is of elliptic type. Although we have demonstrated that the 

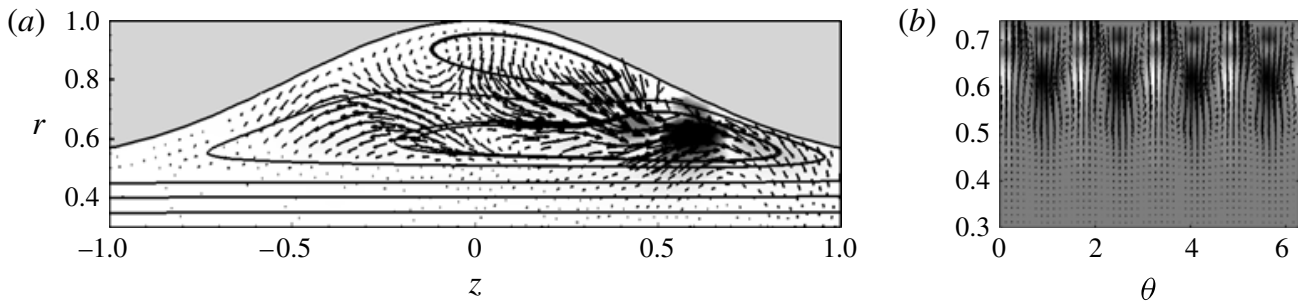

FIGURE 14. (a) Base flow streamlines (continuous lines), critical velocity fields (arrows) and total local energy production $\sum_{i=1}^{4} E_{p, i}$. (b) Critical velocity fields (arrows) and the total local energy production $\sum_{i=1}^{4} E_{p, i}$ (grey shading) at an axial location where the energy transfer reaches its maximum. The parameter settings are $H=0.5, R e=5500$ and $m=4$.

instability mechanism is of an elliptic nature at $H=0.5$, the exact regimes where the lift-up mechanism or elliptic mechanism dominate cannot be established rigorously, and the value of the bulge height at which the transition occurs is not well-defined.

\section{Conclusion}

It may be tempting to draw conclusions concerning the pathophysiology of abdominal aortic aneurysms from the results found in this study. However, in our opinion this idealized model is too far removed from reality to allow one to draw such conclusions. The motivation for the present study is to provide a basis for further, more realistic studies, which should enable us to quantify the effects of pulsatility of the flow (as will be done in a sequel to this paper), non-axisymmetry and other characteristics of the geometry of aneurysms, as well as the presence of the iliac bifurcation and other vasculature downstream of the aneurysm. Nevertheless, the present study does suggest that in inflated arteries, regions of slowly recirculating fluid occur, with very low WSS, which alternate in direction during a flow pulsation.

The steady axisymmetric flow through the model aneurysm studied here is unstable for Reynolds numbers that correspond to the peak values observed under physiological conditions. This instability occurs for dimensionless aneurysm 'heights' of approximately $H=0.3$. Also, the flow in relatively shallow aneurysms (i.e. with large widths $W$ ) is more susceptible to instability than that through more localized aneurysms. The base flow in these shallow aneurysms is characterized by a single large recirculation region; this flow becomes unstable to a stationary mode by the lift-up mechanism. Steady flows through more localized aneurysms also include a secondary recirculation zone; these flows become unstable to oscillatory modes which involve energy transfer from the base flow to perturbations in a small region at the downstream end of the aneurysm, presumably by an elliptical instability mechanism.

\section{REFERENCES}

Blackburn, H. M. \& SHerwin, S. J. 2007 Instability modes and transition of pulsatile stenotic flow: pulse-period dependence. J. Fluid Mech. 573, 57-88.

Blackburn, H. M., Sherwin, S. J. \& Barkley, D. 2008 Convective instability and transient growth in steady and pulsatile stenotic flows. J. Fluid Mech. 607, 267-277.

Bluestein, D., Niu, L., Schoephoerster, R. T. \& Dewanjee, M. K. 1996 Steady flow in an aneurysm model: correlation between fluid dynamics and blood platelet deposition. Trans. ASME: J. Biomed. Engng 118, 280-286. 
Boiron, O., Deplano, V. \& Pelissier, R. 2007 Experimental and numerical studies on the starting effect on the secondary flow in a bend. J. Fluid Mech. 574, 109-129.

Budwig, R., Elger, D., Hooper, H. \& Slippy, J. 1993 Steady flow in abdominal aortic aneurysm models. Trans. AMSE: J. Biomed. Engng 115, 418-423.

Ehrenstein, U. \& Gallaire, F. 2005 On two-dimensional temporal modes in spatially evolving open flows: the flat-plate boundary layer. J. Fluid Mech. 536, 221-233.

FInOL, E. A. \& AMON, C. H. 2001 Blood flow in abdominal aortic aneurysms: pulsatile flow haemodynamics. Trans. ASME: J. Biomech. Engng 123, 474-484.

FInOL, E. A. \& AMON, C. H. $2002 a$ Flow-induced wall shear stress in abdominal aortic aneurysm: part I - steady flow haemodynamics. Comput. Meth. Biomech. Biomed. Engng 5, 309-318.

FInOL, E. A. \& AMON, C. H. $2002 b$ Flow-induced wall shear stress in abdominal aortic aneurysm: part II - pulsatile flow haemodynamics. Comput. Meth. Biomech. Biomed. Engng 5, 319-328.

Gopalakrishnan, S. S. 2014 Dynamics and stability of flow through an abdominal aortic aneurysm. $\mathrm{PhD}$ thesis, Université de Lyon, http://hal.archives-ouvertes.fr/tel-00954202.

Griffith, M. D., Leweke, T., Thompson, M. C. \& Hourigan, K. 2008 Steady inlet flow in stenotic geometries: convective and absolute instabilities. J. Fluid Mech. 616, 111-133.

Griffith, M. D., Leweke, T., Thompson, M. C. \& Hourigan, K. 2009 Pulsatile flow in stenotic geometries: flow behaviour and stability. J. Fluid Mech. 622, 291-320.

Griffith, M. D., Leweke, T., Thompson, M. C. \& Hourigan, K. 2013 Effect of small asymmetries on axisymmetric stenotic flow. J. Fluid Mech. 721, R1.

Griffith, M. D., Thompson, M. C., Leweke, T., Hourigan, K. \& Anderson, W. P. 2007 Wake behaviour and instability of flow through a partially blocked channel. J. Fluid Mech. 582, 319-340.

Humphrey, J. D. \& TAYlor, C. A. 2008 Intracranial and abdominal aortic aneurysms: similarities, differences, and need for a new class of computational models. Annu. Rev. Biomed. Engng 10, 221-246.

KU, D. N. 1997 Blood flow in arteries. Annu. Rev. Fluid Mech. 29, 399-434.

Lanzerstorfer, D. \& Kuhlmann, H. C. 2012 Global stability of the two-dimensional flow over a backward-facing step. J. Fluid Mech. 693, 1-27.

Lasheras, J. C. 2007 The biomechanics of arterial aneurysms. Annu. Rev. Fluid Mech. 39, 293-319.

Mao, X., Sherwin, S. J. \& Blackburn, H. M. 2011 Transient growth and bypass transition in stenotic flow with a physiological waveform. Theor. Comput. Fluid Dyn. 25, 31-42.

Marquet, O., Lombardi, M., Chomaz, J. M., Sipp, D. \& Jacquin, L. 2009 Direct and adjoint global modes of a recirculation bubble. J. Fluid Mech. 622, 1-21.

NAyAR, N. \& ORTEGA, J. M. 1993 Computation of selected eignevalues of generalized eigenvalue problems. J. Comput. Phys. 108, 8-14.

Nerem, R. M., SeED, W. A.\& Wood, N. B. 1972 An experimental study of the velocity distribution and transition to turbulence in the aorta. J. Fluid Mech. 52, 137-160.

Peattie, R. A., Asbury, C. L., Bluth, E. I. \& Ruberti, J. W. 1996 Steady flow in models of abdominal aortic aneurysms. Part I: investigation of the velocity patterns. J. Ultrasound Med. 15, 679-688.

Peattie, R. A., Schrader, T., Bluth, E. I. \& Comstock, C. E. 1994 Development of turbulence in steady flow through models of abdominal aortic aneurysms. J. Ultrasound Med. 13, 467-472.

Peterson, S. D. \& Plesniak, M. 2008 The influence of inlet velocity profile and secondary flow on pulsatile flow in a model artery with stenosis. J. Fluid Mech. 616, 263-301.

SAlsaC, A.-V., Sparks, S. R., ChomaZ, J.-M. \& Lasheras, J. C. 2006 Evolution of the wall shear stresses during the progressive enlargement of symmetric abdominal aortic aneurysms. J. Fluid Mech. 560, 19-51.

ShEARD, G. J. 2009 Flow dynamics and wall shear stress variation in a fusiform aneurysm. J. Engng Maths 64, 379-390.

Sherwin, S. J. \& Blackburn, H. M. 2005 Three-dimensional instabilities and transition of steady and pulsatile axisymmetric stenotic flows. J. Fluid Mech. 533, 297-327.

Shortis, T. A. \& HALL, P. 1999 On the nonlinear stability of the oscillatory viscous flow of an incompressible fluid in a curved pipe. J. Fluid Mech. 379, 145-163. 
Sorensen, D. C. 1992 Implicit application of polynomial filters in a $k$-step Arnoldi method. SIAM J. Matrix Anal. Applics. 13, 357-385.

Theofilis, V. 2011 Global linear instability. Annu. Rev. Fluid Mech. 43, 319-352.

Trip, R., Kuik, D. J., Westerweel, J. \& Poelma, C. 2012 An experimental study of transitional pulsatile pipe flow. Phys. Fluids 24, 014103.

Vétel, J., Garon, A., Pelletier, D. \& Farinas, M. I. 2008 Asymmetry and transition to turbulence in a smooth axisymmetric constriction. J. Fluid Mech. 607, 351-386.

YIP, T. H. \& YU, S. C. M. 2001 Cyclic transition to turbulence in rigid abdominal aortic aneurysm models. Fluid Dyn. Res. 29, 81-113. 